\title{
Saúde sexual e reprodutiva juvenil: reflexões na Atenção Primária à Saúde
}

\author{
Sexual and youth reproductive health: reflections in Primary Health Care \\ Salud sexual y reproductiva de los jóvenes: reflexiones sobre la Atención Primaria de Salud
}

Recebido: 10/02/2022 | Revisado: 18/02/2022 | Aceito: 21/02/2022 | Publicado: 03/03/2022

Kalyne Araújo Bezerra

ORCID: https://orcid.org/0000-0001-8108-9980 Universidade Federal do Rio Grande do Norte, Brasil

E-mail: kalyneearaujo@gmail.com

Pollyanna Jorge Canuto

ORCID: https://orcid.org/0000-0003-0617-9008 Universidade Federal da Bahia, Brasil E-mail: pollyannacanuto@hotmail.com

Cinthia Sonaly Santos Rodrigues

ORCID: https://orcid.org/0000-0002-4465-7640 Universidade Estadual da Paraíba, Brasil E-mail: cinthia6856@gmail.com

\begin{abstract}
Resumo
Objetivo: Identificar as reflexões acerca da saúde sexual e reprodutiva de jovens no âmbito da Atenção Primária à Saúde. Método: A pesquisa foi elaborada por meio de uma revisão integrativa da literatura, de abordagem descritiva. A busca foi realizada na Biblioteca Virtual em Saúde com os descritores registrados no Descritores em Ciências da Saúde: "adolescente", "atenção primária à saúde" e "saúde sexual e reprodutiva" e na PubMed Central com os descritores do Medical Subject Headings: "adolescent", "primary health care" e a palavra-chave "sexual and reproductive health", em ambas as plataformas utilizou-se o operador booleano "AND” obtendo como resultado 801 publicações na busca inicial. Foram incluídos documentos com texto completo, publicados nos últimos 5 anos, nos idiomas português, inglês e espanhol. Após a leitura selecionou-se 14 documentos científicos, porém 2 foram excluídos pois encontravam-se duplicados. Os dados foram analisados e organizados em planilha no Microsoft Excel. Resultados: Conforme os achados, a avaliação dos serviços prestados e a qualidade dos cuidados acerca da saúde sexual e reprodutiva, a visão dos profissionais sobre a temática e a acessibilidade apresentaram-se como as principais perspectivas de atuação na Atenção Primária à Saúde para os jovens. Conclusão: Portanto, a saúde sexual e reprodutiva dos adolescentes é de relevância peculiar, dada aos seus exequíveis desfechos na saúde a curto e longo prazo.
\end{abstract}

Palavras-chave: Atenção primária à saúde; Políticas públicas de saúde; Adolescente; Saúde sexual; Saúde reprodutiva.

\begin{abstract}
Objective: To identify reflections on the sexual and reproductive health of young people in the context of Primary Health Care. Method: The research was carried out through an integrative literature review, with a descriptive approach. The search was performed in the Virtual Health Library with the descriptors registered in the Health Sciences Descriptors: "adolescent", "primary health care" and "sexual and reproductive health" and in PubMed Central with the descriptors of the Medical Subject Headings: " adolescent", "primary health care" and the keyword "sexual and reproductive health", in both platforms the Boolean operator "AND" was used, resulting in 801 publications in the initial search. Full-text documents published in the last 5 years in Portuguese, English and Spanish were included. After reading, 14 scientific documents were selected, but 2 were excluded because they were duplicates. Data were analyzed and organized in a Microsoft Excel spreadsheet. Results: According to the findings, the evaluation of the services provided and the quality of care regarding sexual and reproductive health, the professionals' view on the subject and accessibility were presented as the main perspectives of action in Primary Health Care for young people. Conclusion: Therefore, the sexual and reproductive health of adolescents is of particular relevance, given its feasible short- and long-term health outcomes.
\end{abstract}

Keywords: Primary health care; Public health policies; Adolescent; Sexual health; Reproductive health.

\section{Resumen}

Objetivo: Identificar reflexiones sobre la salud sexual y reproductiva de los jóvenes en el contexto de la Atención Primaria de Salud. Método: La investigación se realizó a través de una revisión integrativa de la literatura, con enfoque descriptivo. La búsqueda se realizó en la Biblioteca Virtual en Salud con los descriptores registrados en los Descriptores de Ciencias de la Salud: "adolescente", "atención primaria en salud" y "salud sexual y reproductiva" y en PubMed Central con los descriptores de los Medical Subject Headings: "adolescente", "atención primaria en salud" 
y la palabra clave "salud sexual y reproductiva", en ambas plataformas se utilizó el operador booleano "AND", resultando 801 publicaciones en la búsqueda inicial. Se incluyeron documentos a texto completo publicados en los últimos 5 años en portugués, inglés y español. Después de la lectura, se seleccionaron 14 documentos científicos, pero 2 fueron excluidos por ser duplicados. Los datos fueron analizados y organizados en una hoja de cálculo de Microsoft Excel. Resultados: De acuerdo con los hallazgos, la evaluación de los servicios prestados y la calidad de la atención en salud sexual y reproductiva, la visión de los profesionales sobre el tema y la accesibilidad se presentaron como las principales perspectivas de actuación en la Atención Primaria de Salud de los jóvenes. Conclusión: Por lo tanto, la salud sexual y reproductiva de los adolescentes es de particular relevancia, dados sus resultados de salud factibles a corto y largo plazo.

Palabras clave: Primeros auxilios; Políticas de salud pública; Adolescente; Salud sexual; Salud reproductiva.

\section{Introdução}

Os adolescentes e jovens correspondem a 1,8 bilhão da população mundial e no Brasil, representam $30 \%$ dos habitantes. De acordo com a OMS (Organização Mundial de Saúde), compreende-se como adolescentes, a faixa etária entre 10 e 19 anos e jovens, de 15 a 24 anos (ONU, 2014).

A adolescência é um período de descobertas e novas experiências, estando entre estas, a iniciação da atividade sexual, que tem sido evidenciada de forma cada vez mais precoce entre estes indivíduos (OPAS, 2017). Nessa fase, a experiência com a sexualidade e a materialização das práticas sexuais associadas à falta de percepção dos adolescentes com a maturidade para vivenciar este momento, tornam estes indivíduos mais suscetíveis ao risco de desenvolvimento de Infecções Sexualmente Transmissíveis (ISTs), bem como de gestações não planejadas (Franco et al., 2020).

No Brasil, 21,3\% dos nascimentos no ano de 2007 foram relativos às mães com idade entre 10 e 19 anos (WHO, 2015). Dados da OMS apontam que o Brasil configura-se como um dos sete países em que metade de todos os nascimentos, são de mães adolescentes (Ministério da Saúde, 2008). Além disso, cabe destacar que no ano de 2012, a primeira causa de internação hospitalar referente ao sexo feminino, no Brasil, correspondeu ao parto na faixa etária entre 10 e 19 anos (Ministério da Saúde, 2013).

Com estimativa de 340 milhões novos casos por ano, as ISTs entre os adolescentes, constituem um problema de saúde pública à nível mundial. Estes índices elevados, associados ao elevado número de partos na adolescência e ao início precoce das relações sexuais, apontam para a necessidade de uma assistência adequada e que atenda às necessidades desta população (Gondim et al., 2015).

A saúde sexual consiste em bem-estar físico, emocional, mental e social que deve ser abordado de forma respeitosa e positiva. A saúde reprodutiva é uma ação da atenção básica realizada por meio de atividades educativas, aconselhamento e atividades clínicas, incluindo nestas, o planejamento reprodutivo que contribui para uma prática sexual saudável. Vale ressaltar que a prática das atividades educativas deve ser realizada nos locais de inserção dos adolescentes e jovens, como nos domicílios, escolas e espaços comunitários, atentando para a confidencialidade (Brasil, 2013).

Apesar de ser pontuada como atividade da atenção básica no que tange à saúde do adolescente, a Saúde Sexual e Reprodutiva (SSR) ainda é apontada como frágil nestes serviços. Um estudo realizado em 70 países classificados como de baixa e média renda, observou que, em sua maioria, apenas $10 \%$ de todos os adolescentes, visitaram uma unidade de saúde nos últimos 12 meses e receberam orientações voltadas ao planejamento familiar e SSR (Woog et al., 2015).

Em outro estudo realizado em unidades ambulatoriais do município do Rio de Janeiro, constatou-se que, das 229 unidades que prestavam o atendimento ambulatorial no Serviço Único de Saúde - SUS, apenas 148 ofereciam serviços relativos à SSR. Relacionado às atividades educativas voltadas para os jovens, apenas 12,9\% das unidades realizavam esse tipo de serviço e apontavam como barreiras para a baixa realização, a falta de capacitação e precariedade de recursos humanos (Taquette et al., 2017). 
O desenvolvimento de ações de educação sexual, se estabelece como um forte aliado quando realizado, principalmente, antes da primeira relação sexual, enfatizando reflexões que ajudem nas tomadas de decisões relacionadas à sexualidade e vida reprodutiva, abordando conhecimentos e desenvolvendo ações que propiciem conhecimento aos jovens sobre os riscos de ISTs, enfatizando os métodos de prevenção (Brasil, 2010).

Nesse contexto, a Atenção Primária à Saúde - APS, possui papel fundamental no que tange a SSR de adolescentes e jovens, sendo as ações de saúde sexual e reprodutiva, um dos eixos prioritários deste serviço. Além disso, as atividades desenvolvidas pela APS, devem compreender a assistência clínica e o aconselhamento, estendendo-se de forma longitudinal ao pré-natal, parto e puerpério, atendimento às ISTs e outras afecções do aparelho reprodutor, fortalecimento do exercício da paternidade responsável, encaminhamento e orientações ao tratamento apropriado, em casos de infertilidade entre outros (Nasser et al., 2017; Paiva, Caetano, 2020).

Para tanto, profissionais de saúde atuantes na APS, destacam competências necessárias para a realização da SSR de qualidade para adolescentes e jovens nestes serviços, como a escuta ativa, o diálogo, o não julgamento e não preconceito, a empatia e o sigilo profissional. Porém, ao observar a existência do preconceito e do tabu por parte dos próprios profissionais atuantes, a implementação da SSR torna-se dificultosa (Telo; Eitt, 2018), destacando que a abordagem de assuntos relacionados aos métodos contraceptivos, sexualidade e ISTs, causam constrangimento (Maranhão et al., 2017) e que boa parte dos profissionais não se sentem preparados para abordá-los (Brasil, 2013).

Desta forma, destaca-se uma pesquisa realizada no estado do Piaú, no Nordeste brasileiro, na qual $72,2 \%$ dos jovens participantes receberam informações a respeito da prevenção a ISTs e gravidez, no ambiente escolar, e 42,5\% pelos pais. Além disso, mais da metade dos jovens, não procuraram orientações nos serviços de saúde antes da primeira relação sexual (Brasil, 2013). Em outro estudo, as informações sobre tal, são buscadas inicialmente com amigos, pais, familiares e, em última circunstância, procuram os professores e serviços de saúde (Maranhão et al., 2017).

Nesse contexto, abordar a sexualidade tendo os adolescentes como público-alvo, é de fundamental importância para ajudá-los a entender a ocorrência de mudanças corporais, os sentimentos e aflições gerados nessa fase, e com isso, buscar medidas eficazes na promoção à SSR destes indivíduos (Brasil, 2013). Dado o exposto, surgem as seguintes problemáticas: "Quais as dificuldades encontradas na promoção à SSR de adolescentes e jovens na APS?" e "Quais as reflexões e abordagens destacadas pelos profissionais da APS no que tange à SSR de adolescentes e jovens?”. Em vista disso, o presente estudo teve como objetivo, identificar as dificuldades e reflexões acerca da saúde sexual e reprodutiva juvenil no âmbito da Atenção Primária à Saúde na literatura científica.

\section{Metodologia}

A pesquisa refere-se a uma revisão integrativa da literatura do tipo descritiva de abordagem qualitativa, a fim de responder à questão norteadora seguindo as seis etapas de tal método, sendo elas: 1. Elaboração da questão norteadora; 2. Definição de critérios de inclusão e exclusão; 3. Definição das informações extraídas dos estudos; 4. Avaliação dos estudos da amostra; 5. Interpretação dos resultados; 6. Apresentação da revisão (Sousa et al., 2017).

A busca foi realizada em dois momentos. Inicialmente aplicou-se os descritores registrados nos Descritores em Ciências da Saúde - DeCS, sendo eles: "adolescente", "atenção primária à saúde" e "saúde sexual e reprodutiva", combinados pelo operador booleano "AND” na Biblioteca Virtual em Saúde - BVS. Na busca inicial, obteve-se como resultado, 219 publicações. Em seguida, foram aplicados os filtros de textos completos e disponíveis, publicados nos últimos cinco anos (2015 a 2020) em português, inglês e espanhol, resultando em um total de 56 documentos científicos encontrados. Após leitura de títulos, resumos e textos completos, a amostra totalizou 8 artigos. 
Posteriormente, foram aplicados os descritores registrados no MeSH Database: "adolescent", "primary health care" e a palavra-chave "sexual and reproductive health", combinados pelo operador booleano "AND" na base de dados PubMed Central - PMC. Na busca inicial foram obtidos 582 documentos científicos. Após a aplicação dos filtros, restaram 125 artigos, com a leitura dos títulos, resumos e texto completo, a amostra totalizou 6 documentos.

Ao final, foram selecionadas 14 publicações, porém duas destas encontravam-se duplicadas nas bases de dados, perfazendo um total de 12 artigos selecionados para compor o presente estudo, conforme o mostra o fluxograma (Figura 1). A amostra foi analisada e foram extraídos os dados referentes ao método de estudo, classificação do nível de evidência, título, objetivo e as dificuldades e abordagens em planilhas no programa do Microsoft Excel versão 2019. Em seguida, os dados foram agrupados em um quadro descritivo (Quadro 1).

Figura 1 - Fluxograma de identificação e seleção dos estudos.

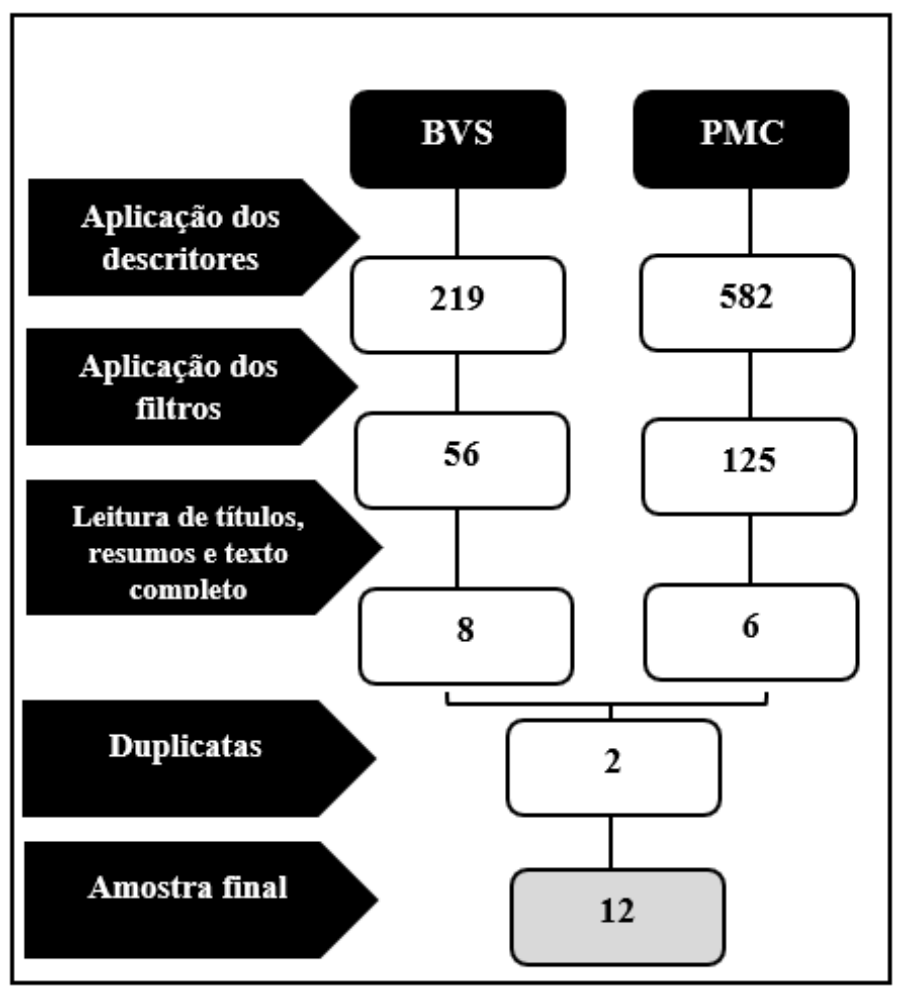

Fonte: Autoria própria (2020).

\section{Resultados}

Após a definição da amostra, os 12 documentos da amostra foram analisados para a extração de informações pertinentes quanto à pergunta de pesquisa. Em seguida, as mesmas foram categorizadas quanto ao método de estudo e classificação do nível de evidência (Stillwell et al., 2020), título, objetivo do estudo e as dificuldades e abordagens. 
Quadro 1 - Descrição dos documentos quanto ao método de estudo e nível de evidência, título, objetivo e as dificuldades e abordagens $(n=12)$.

\begin{tabular}{|c|c|c|c|}
\hline $\begin{array}{l}\text { Método/ nível } \\
\text { de evidência }\end{array}$ & Título & Objetivo & Considerações acerca das abordagens \\
\hline $\begin{array}{l}\text { Estudo } \\
\text { transversal/ } \\
\text { Nível VI }\end{array}$ & $\begin{array}{l}\text { Do European Union countries } \\
\text { adequately address the healthcare } \\
\text { needs of adolescents in the area of } \\
\text { sexual reproductive health and rights? }\end{array}$ & $\begin{array}{l}\text { Avaliar recomendações e políticas } \\
\text { relacionadas ao acesso à assistência na área } \\
\text { de saúde e direitos sexuais e reprodutivos } \\
\text { dos adolescentes em } 31 \text { países europeus } \\
\text { (União Européia (UE) mais Islândia, } \\
\text { Noruega e Suíça). }\end{array}$ & $\begin{array}{l}18 \text { países da União Europeia apresentaram serviços } \\
\text { de atenção especializada aos adolescentes que, em } \\
\text { sua maioria prestam serviços de saúde sexual e } \\
\text { reprodutiva; } 10 \text { países não elaboraram políticas de } \\
\text { confidencialidade. }\end{array}$ \\
\hline $\begin{array}{l}\text { Estudo } \\
\text { transversal/ } \\
\text { Nível VI }\end{array}$ & $\begin{array}{l}\text { Knowledge and Acceptability of } \\
\text { Long-Acting } \\
\text { Contraception Among Adolescent } \\
\text { Women Receiving School-Based } \\
\text { Primary Care Services. }\end{array}$ & $\begin{array}{l}\text { Avaliar correlatos de conhecimento e } \\
\text { aceitabilidade } \\
\text { dos métodos contraceptivos reversíveis de } \\
\text { ação prolongada entre mulheres } \\
\text { adolescentes em um centro de saúde } \\
\text { escolar. }\end{array}$ & $\begin{array}{l}\text { Baixo nível de conhecimento geral sobre os } \\
\text { métodos, encontrados principalmente entre } \\
\text { mulheres brancas jovens ou que iniciaram a vida } \\
\text { sexual. }\end{array}$ \\
\hline $\begin{array}{l}\text { Estudo } \\
\text { transversal/ } \\
\text { Nível VI }\end{array}$ & $\begin{array}{l}\text { Knowledge, utilization and barriers to } \\
\text { primary care services for sexual and } \\
\text { reproductive health among } \\
\text { adolescents in secondary schools in } \\
\text { Selangor, Malaysia. }\end{array}$ & $\begin{array}{l}\text { Examinar o conhecimento, a utilização e as } \\
\text { barreiras dos adolescentes malaios aos } \\
\text { serviços de atenção primária à saúde sexual } \\
\text { e reprodutiva. }\end{array}$ & $\begin{array}{l}72,3 \% \text { dos adolescentes não procuram o serviço de } \\
\text { saúde sexual e reprodutiva por não se sentirem } \\
\text { confortáveis, devido a vergonha, a preocupação } \\
\text { com confidencialidade, a hostilidade da equipe de } \\
\text { saúde na visão dos adolescentes e os custos } \\
\text { decorrentes da assistência. }\end{array}$ \\
\hline $\begin{array}{l}\text { Estudo } \\
\text { transversal/ } \\
\text { Nível VI }\end{array}$ & $\begin{array}{l}\text { Assessment of adolescent and youth } \\
\text { friendly services in primary healthcare } \\
\text { facilities in two provinces in South } \\
\text { Africa. }\end{array}$ & $\begin{array}{l}\text { Detalhar a avaliação do serviço amigável } \\
\text { para adolescentes e jovens em relação aos } \\
\text { padrões definidos para informar iniciativas } \\
\text { para fortalecer esses serviços. }\end{array}$ & $\begin{array}{l}\text { Serviços de saúde com oferta de testes para HIV, } \\
\text { planejamento familiar, pré-natal e gerenciamento } \\
\text { de infecções sexualmente transmissíveis. }\end{array}$ \\
\hline $\begin{array}{l}\text { Estudo } \\
\text { transversal/ } \\
\text { Nível VI }\end{array}$ & $\begin{array}{l}\text { Healthcare workers' beliefs, } \\
\text { motivations and behaviours affecting } \\
\text { adequate provision of sexual and } \\
\text { reproductive healthcare services to } \\
\text { adolescents in Cape Town, South } \\
\text { Africa: a qualitative study. }\end{array}$ & $\begin{array}{l}\text { Explorar e obter uma } \\
\text { compreensão aprofundada das crenças, } \\
\text { motivações e comportamentos dos } \\
\text { profissionais de saúde que afetam a } \\
\text { provisão adequada desses serviços a } \\
\text { adolescentes na África do Sul. }\end{array}$ & $\begin{array}{l}\text { Profissionais desafiados a prestar assistência pela } \\
\text { falta de tempo enquanto que, são motivados pela } \\
\text { preocupação relacionada às altas taxas de gravidez } \\
\text { na adolescência. }\end{array}$ \\
\hline $\begin{array}{l}\text { Estudo } \\
\text { transversal/ } \\
\text { Nível VI }\end{array}$ & $\begin{array}{l}\text { Quality of reproductive healthcare for } \\
\text { adolescents: A nationally } \\
\text { representative survey of providers in } \\
\text { Mexico. }\end{array}$ & $\begin{array}{l}\text { Gerar evidências sobre a qualidade da } \\
\text { assistência em serviços públicos de saúde } \\
\text { sexual e reprodutiva para adolescentes. }\end{array}$ & $\begin{array}{l}\text { Cerca de } 13,9 \% \text { dos serviços oferecem assistência } \\
\text { de baixa qualidade, } 68,6 \% \text { média qualidade e } \\
17,5 \% \text { qualidade alta; unidades com espaço de } \\
\text { atendimento aos adolescentes possuem chances de } \\
\text { oferecer uma alta qualidade. }\end{array}$ \\
\hline $\begin{array}{l}\text { Estudo } \\
\text { transversal/ } \\
\text { Nível VI }\end{array}$ & $\begin{array}{l}\text { Adolescent sexual and reproductive } \\
\text { health: } \\
\text { perceptions of nursing professionals. }\end{array}$ & $\begin{array}{l}\text { Conhecer como os profissionais de } \\
\text { enfermagem da atenção básica à saúde } \\
\text { percebem e abordam a saúde sexual e } \\
\text { reprodutiva dos adolescentes. }\end{array}$ & $\begin{array}{l}\text { Para os enfermeiros, a sexualidade é fator para o } \\
\text { desenvolvimento da autonomia e a saúde sexual e } \\
\text { reprodutiva atua como educação preventiva. }\end{array}$ \\
\hline $\begin{array}{l}\text { Estudo } \\
\text { transversal/ } \\
\text { Nível VI }\end{array}$ & $\begin{array}{l}\text { Serbian primary care physicians' } \\
\text { perspectives on adolescents' right to } \\
\text { confidentiality in sexual and } \\
\text { reproductive healthcare-a qualitative } \\
\text { interview study. }\end{array}$ & $\begin{array}{l}\text { Avaliar as perspectivas dos médicos da } \\
\text { atenção primária sobre o direito legal dos } \\
\text { menores a confidencialidade nos cuidados } \\
\text { de saúde sexual e reprodutiva, bem como } \\
\text { suas experiências na }\end{array}$ & $\begin{array}{l}\text { A confidencialidade é prejudicada devido a falta } \\
\text { de médicos. }\end{array}$ \\
\hline $\begin{array}{l}\text { Estudo } \\
\text { transversal/ } \\
\text { Nível VI }\end{array}$ & $\begin{array}{l}\text { Substantiating the need for primary } \\
\text { care-based sexual health promotion } \\
\text { interventions for ethnic minority } \\
\text { adolescent women experiencing } \\
\text { health disparities. }\end{array}$ & $\begin{array}{l}\text { Avaliar o contexto de sofrimento } \\
\text { psicológico, violência e uso de substâncias } \\
\text { entre mulheres adolescentes africanas e } \\
\text { mexicanas-americanas com histórico de } \\
\text { IST, violência ou comportamento de alto }\end{array}$ & $\begin{array}{l}\text { Integrar serviços de promoção a saúde sexual } \\
\text { levando considerando o histórico de HIV, } \\
\text { comportamento e risco de infecção por infecções } \\
\text { sexualmente transmissíveis afim de realizar } \\
\text { intervenções diretas. }\end{array}$ \\
\hline $\begin{array}{l}\text { Estudo } \\
\text { transversal/ } \\
\text { Nível VI }\end{array}$ & $\begin{array}{l}\text { Accessibility of adolescents to sources } \\
\text { of information on } \\
\text { sexual and reproductive } \\
\text { health. }\end{array}$ & $\begin{array}{l}\text { Identificar as fontes de informações } \\
\text { utilizadas pelos adolescentes da escola } \\
\text { pública sobre sexualidade e reprodução. }\end{array}$ & $\begin{array}{l}\text { Realização de atividades educativas sobre saúde } \\
\text { sexual e reprodutiva encontra-se a escola e a } \\
\text { igreja; procuram os amigos para tirar suas dúvidas } \\
\text { e buscam informações na televisão, internet, livros } \\
\text { e revistas. }\end{array}$ \\
\hline $\begin{array}{l}\text { Estudo } \\
\text { transversal/ } \\
\text { Nível VI }\end{array}$ & $\begin{array}{l}\text { Sexual and Reproductive Health Care } \\
\text { Receipt Among Young Males Aged } \\
\text { 15-24. }\end{array}$ & $\begin{array}{l}\text { Descrever o recebimento de cuidados } \\
\text { sexuais e de saúde reprodutiva de homens } \\
\text { jovens por comportamento sexual e fatores } \\
\text { associados a um maior recebimento desses } \\
\text { cuidados. }\end{array}$ & $\begin{array}{l}\text { Poucos jovens que receberam cuidados de saúde } \\
\text { sexual e reprodutiva, principalmente com vida } \\
\text { sexual ativa. }\end{array}$ \\
\hline $\begin{array}{l}\text { Caderno de } \\
\text { Saúde da } \\
\text { Família }\end{array}$ & $\begin{array}{l}\text { Saúde do adolescente e a saúde da } \\
\text { família }\end{array}$ & $\begin{array}{l}\text { Discutir o papel dos profissionais de saúde } \\
\text { nas ações e estratégias voltadas à saúde dos } \\
\text { adolescentes, bem como abordar situações } \\
\text { clínicas e agravos específicos desse ciclo de } \\
\text { vida. }\end{array}$ & $\begin{array}{l}\text { Atentar para as necessidades dos adolescentes } \\
\text { como: início precoce das atividades sexuais e uso } \\
\text { de métodos contraceptivos errôneo, sabendo que as } \\
\text { meninas se preocupam com gravidez e os meninos } \\
\text { com a contaminação de infecções sexualmente } \\
\text { transmissíveis. }\end{array}$ \\
\hline
\end{tabular}

Fonte: Dados da pesquisa (2020). 


\section{Discussão}

O âmbito da SSR na adolescência precisa agregar-se a noções ampliadas de saúde, onde suas necessidades devem ser priorizadas globalmente, e, infelizmente, muitas dessas premências permanecem incipientes. Logo, este estudo evidenciou algumas considerações perante esta premissa.

Sobre a SSR e a saúde dos adolescentes, de uma maneira ampla, um estudo detalhou sobre o atendimento na União Europeia neste atente, inferiu que mais de $30 \%$ dos países investigados não possuíam políticas de confidencialidade para atender ao adolescente, assim como quase metade desta amostra também não possuíam centros de especialidades (Michaud et al., 2020). Outra pesquisa (Champion et al., 2016), corrobora com estes achados referentes a falta de acessibilidade e confidencialidade interferentes na promoção da SSR dos adolescentes.

Por esta problemática, remete-se que são poucas as políticas voltadas a SSR, raros serviços direcionados e baixa acessibilidade aos jovens, sendo em muitos locais evidenciado apenas a procura por métodos de contracepção de emergência, tornando assim, não condizente com as práticas ideais de prevenção e promoção da saúde, ambas inerentes a APS. Em um dos achados (Pinho, Garcia, 2016), ficou nítido que o acesso a serviços de saúde possuem acolhimento das necessidades, de forma eficaz na integralidade da atenção à saúde e nas especificidades que essa faixa etária exige, sendo imprescindível para a efetivação das diretrizes dispostas na Política Nacional de Atenção Integral à Saúde de Adolescentes e Jovens.

Em contrapartida, quando envolvem avaliações dos serviços, apontamentos sobre a direção da assistência no tocante a SSR, vislumbram a garantia da saúde dos adolescentes, especialmente na prevenção de gestações indesejadas e ISTs, além de incrementar no manejo psicossocial como abordagem imprescindível. Destarte, o fortalecimento das atividades se concentra no treinamento dos profissionais de saúde, que inclui à melhoria do atendimento clínico, psicossocial e gerencial quanto aos adolescentes, além da condução de grupos de apoio e instituição de cronogramas de acesso aos cuidados e possíveis tratamentos (James et al., 2018).

Singularmente, diante da visão dos profissionais de saúde sobre a abordagem a SSR se caracteriza como ampla e oportuna, uma vez que ressalta a atuação dos mesmos na APS congregando sobre o aporte e as práxis pertinentes ao processo de cuidar holístico dos jovens. Na perspectiva dos médicos Sérvios, a maioria apoia a confidencialidade como caráter relevante no processo, reconhecendo sua falta como impedimento no acesso dos adolescentes aos serviços (Stojkovic et al., 2019).

Por outro lado, os profissionais de enfermagem denotaram um outro enfoque sobre a SSR e suas concepções. Num estudo africano, as crenças, motivações e comportamentos afetaram a prestação de cuidados na SSR de adolescentes, pois alguns alegaram habilidades insuficientes, dificultando a prestação dos serviços adequados, e acesso limitado às escolas para educação em saúde e prevenção de agravos (Jonas et al., 2018). Outrem, a percepção dos enfermeiros aponta visões reducionistas sobre a SSR na APS, com limitações no entendimento de uma abordagem holística do cuidado e da educação sexual e reprodutiva, inferindo muitos desafios a serem compreendidos (Sehnem et al., 2019).

Ademais, prismas sobre a acessibilidade e o conhecimento dos cuidados em SSR tange relevantes abordagens sobre a tônica. No quesito acesso a estes serviços de SSR, um dos estudos (Godim et al., 2015) evidenciou uma elevada acessibilidade a atividades de educação em SSR, tendo a escola como espaço favorável, sendo a TV a fonte de informação mais procurada, e não a APS. Já em estudo de outro autor (Marcell et al., 2018), dos 427 jovens do sexo masculino, entre 15 a 24 anos, recrutados em 3 unidades de APS, 1 em cada 10 receberam todos os serviços pertinentes à SSR. Na mesma linha, quando de 680 adolescentes de uma pesquisa que avaliou a utilização e as barreiras sobre o acesso, 1 em cada 10 estão cientes dos serviços de SSR na APS, porém apenas 7\% visitaram o mesmo para tal assistência (Othman et al., 2019).

Por tudo isso (Hoopes et al., 2016), o conhecimento e a acessibilidade entre adolescentes que receberam atendimento de APS na escola sobre SSR é assertivo, onde dos 102 estudantes participantes da pesquisa, compreendendo a faixa etária de 14 a 19 anos, a maioria possui conhecimentos sobre a contracepção na escola. Ainda sobre abordagens profícuas, a qualidade 
dos cuidados de SSR para os adolescentes também foram verificados num estudo mexicano. Das 505 unidades, a grande maioria possui uma qualidade moderada (69\%), além disso, as urbanas e metropolitanas possuem 10 vezes maior chance de oferecer atendimentos de alta qualidade, e unidades com espaço para aconselhamento tiveram pelo menos 8 vezes maiores chances de prestar assistência de alta qualidade (Villalobos et al., 2017).

Assim, considerações sobre a SSR dos adolescentes e jovens adultos foram demandadas como axioma relevante na APS, dos quais os estudos apontaram abordagens de conhecimentos, usabilidade dos serviços e percepções sobre o contexto expressivo de promoção à saúde e prevenção de doenças na saúde juvenil.

\section{Conclusão}

Sobre as reflexões deste estudo, a SSR imanente a APS fora abordada de maneira substancial, desde a avaliação dos serviços, passando pela visão dos profissionais sobre o assunto, até a acessibilidade na assistência, a qualidade dos cuidados e o conhecimento dos atendimentos ofertados.

Vários estudos demonstraram interessantes abordagens para os adolescentes no que concerne a SSR na APS, de onde manuscritos apontaram informações sobre acesso aos serviços, outrem argumentaram sobre a usabilidade dos mesmos, assim como padrões de qualidade para tal, apoio a confidencialidade no atendimento, entendimento sobre educação em saúde nesta propositura, assimilação quanto a amplitude das ações prestadas e a significância em ofertar prestação dos serviços com qualidade e sapiência.

Todavia, aspectos restritivos na SSR concernente à APS são superficialmente relatados, onde outrora, perspectivas fatigantes e limitadoras emergem como potenciais abordagens obstrutivas, pleiteando assim medidas importantes nesta conjuntura assistencial dos jovens, com sugestões de novos estudos, e vistas ao fortalecimento e melhorias destas considerações sobre a SSR dos adolescentes.

\section{Referências}

Brasil (2010). Diretrizes nacionais para a atenção integral à saúde de adolescentes e jovens na promoção, proteção e recuperação da saúde. https://bvsms.saude.gov.br/bvs/publicacoes/diretrizes_nacionais_atencao_saude_adolescentes_jovens_promocao_saude.pdf.

Brasil (2013). Departamento de Atenção Básica. Saúde sexual e saúde reprodutiva.

Champion, J. D., Young, C., \& Rew, L. (2016). "Substantiating the need for primary care-based sexual health promotion interventions for ethnic minority adolescent women experiencing health disparities". J Am Assoc. Nurse Pract. 28 (9), 487-492.

Franco, M. S., Barreto, M. T. S., Carvalho, J. W., Silva, P. P., Moreira, W. C. Cavalcante, M. C., et al. (2020). "Educação em saúde sexual e reprodutiva do adolescente escolar". Revista de enfermagem UFPE online, 14, 1-8.

Godim, O. S., Souto, N. F., Moreira, C. B., Cruz, M. E. C, \& Caetano, F. H. P. (2015). “Accessibility of adolescents to sources of information on sexual and reproductive health". Journal of human growth and development, 25 (1), 50-54.

Hoopes, A. J., Ahrens, K. Y. M. R., Gilmore, K., Cady, J., Halland, W. L., Oelschlager, A. M. A, \& Prager, S. (2016). "Knowledge and Acceptability of LongActing Reversible Contraception Among Adolescent Women Receiving School-Based Primary Care Services". Journal Of Primary Care Community Health, 7 (3), 165-170.

James, S, Pisa, P. T., Imrie, J., Beery, M. P., Martin, C., Shokosana, C., \& Morethwey, S. D. (2018). "Assessment of adolescent and youth friendly services in primary healthcare facilities in two provinces in South Africa”. BMC Health Serv Res, 8 (809), 1-10.

Jonas, K., Crutzen, R., Krumeich, A., Roman, N., Bornem, B. V., \& Reddy, V. (2018). "Healthcare workers' beliefs, motivations and behaviours affecting adequate provision of sexual and reproductive healthcare services to adolescents in Cape Town, South Africa: a qualitative study". 2018. BMC Health Serv Res, 18 (109), 1-13.

$\&$

Maranhão, T. A., Gomes, K. R. O., Oliveira. D. C., Moita, N. J. M. (2017). "Repercussão da iniciação sexual na vida sexual e reprodutiva de jovens de capital do Nordeste brasileiro". Ciências e saúde coletiva, 22 (12), 4083-4094.

Marcell, A. V., Gibbs, S. E., Pringlim, N. A., Page, K. R., Arrington, R., Jennigs, J. M., Loosier, P. S., \& Dittus, P. J. (2018). "Sexual and Reproductive Health Care Receipt Among Young Males Aged 15-24". Journal of Adolescent Health, 62 (4), 382-389. 
Michaud, P., Visser, A., Vervoort, J., Kochen, P., Reinjneveld, S., Blair, M., Alexander, D., Rigbyr, M., Weber, M., \& Jansen, D. (2020). Do European Union countries adequately address the healthcare needs of adolescents in the area of sexual reproductive health and rights?. Archives of Disease in Childhood, 105 (1), p. 40-46.

Ministério da Saúde (2008). Indicadores e dados básicos: sistema de informações sobre nascidos vivos (SINASC).

Ministério da Saúde (2013). Sistema Nacional de Dados. Estatísticas vitais.

Nações Unidas Brasil (2014). Adolescentes e Jovens são $28 \%$ da população mundial, ONU pede mais investimentos Nações Unidas Brasil. https://nacoesunidas.org/adolescentes-e-jovens-sao-28-da-populacao-mundial-onu-pede-mais-investimentos/.

Nasser, M. A., Nemes, M. I. B., Andrade, M. C., Prado, R. R., \& Castanheira, E. R. L. (2017). “Avaliação na atenção primária paulista: ações incipientes em saúde sexual e reprodutiva". Revista Saúde Pública, 51 (77), 51-77.

Organização Pan-Americana da Saúde (2017). Ministério da Saúde. Saúde e sexualidade de adolescentes. Construindo equidade no SUS. http://bvsms.saude.gov.br/bvs/publicacoes/saude_sexualidade_adolescente_construindo_equidade_sus.pdf.

Othman, S., Kong, S. Z., \& Mohd Mydin, F. H., C. J. (2019). "Knowledge, utilization and barriers to primary care services for sexual and reproductive health among adolescents in secondary schools in Selangor, Malaysia”. Médico Fam da Malásia, 14 (1), 10-17.

Paiva, C. C. N., Caetano, R. (2020). "Evaluation of the implementation of sexual and reproductive health actions in Primary Care: scope review". Esc. Anna Nery, 24 (1), e20190142.

Pinho, J. R. O., \& Garcia, P. T. (2016). "Saúde do Adolescente e Saúde da família”. Cadernos de Saúde da família. https://ares.unasus.gov.br/acervo/html/ARES/7558/1/Livro\%206\%20-

$\% 20$ Sa\%c3\%bade $\% 20$ do $\% 20$ adolescente $\% 20 \mathrm{e} \% 20 \mathrm{a} \% 20 \mathrm{sa} \%$ c3\%bade $\% 20 \mathrm{da} \% 20 \mathrm{fam} \%$ c3\%adlia.pdf.

Sehnem, D., Graciela, C. B. T. T., Lipinski, J. M., Ribeiro, A. C., Wilhelm, L. A., \& Arboit, J. (2019). "Salud sexual y reproductiva de adolescentes: percepciones de los profesionales en enfermeira". Avances en Enfermería, 37 (3), 343-352.

Sousa, L. M. M., Vieira, C. M., Severino, S., \& Antunes, V. (2017). “Metodologia de Revisão Integrativa da Literatura em Enfermagem”. Revista Investigação em enfermagem, 17-26.

Stillwell, S. B., Overholt, E. F., Mazurek, B., \& Williamson, K. M. (2010). "Searching for the Evidence Strategies to help you conduct a successful search". The American Journal of Nursing, 110 (5), 41-47.

Stojkovic, V. J., Bojana, M., \& Karel, T. (2019). "Serbian primary care physicians' perspectives on adolescents' right to confidentiality in sexual and reproductive healthcare - a qualitative interview study". Family Practice, 36 (3), 317-324.

Taquette, S. R., Monteiro, D. L. M., Rodrigues, N. C. P., Rozenberg, R., Menezes, D. C. S. Rodrigues, A. O., et al. (2017). “Saúde sexual e reprodutiva para a população adolescente, Rio de Janeiro, Brasil”. Ciências e saúde coletiva, 22 (6), 1923-1932.

Telo, S. V., Witt, R. R. (2018). "Saúde sexual e reprodutiva: competências da equipe na Atenção Primária à Saúde”. Ciências e saúde coletiva, 23 (11), 3481 3490 .

Villalobos, A., Allen, B., Salazar, A. J., Castro, F. Barrieros, T., Leyva, L. A., \& Rojas, M. R. (2017). "Quality of reproductive healthcare for adolescents: a nationally representative survey of providers in Mexico". Plos One, 12 (3), 1-8.

Woog, V., Singh, S., Browne, A., \& Philbin, J. (2015). “Adolescent Women's need for and use of sexual and reproductive health Services in Developing Countries". https://www.guttmacher.org/sites/default/files/report_pdf/adolescent-srhs-need-developing-countries.pdf.

World Health Organization (2015). Maternal, newborn, child and adolescent health: adolescent pregnancy. http://www.who.int/maternal_child_adolescent/topics/maternal/adolescent_pregnancy/en. 